\title{
Near Real-Time Low Frequency Load Disaggregation
}

\author{
Selim Sahrane, Mourad Haddadi
}

\begin{abstract}
Device-level power consumption information can lead to considerable energy savings. Smart meters are being adopted in several countries, but they are only capable of measuring the total power consumption. NonIntrusive Load Monitoring (NILM) aims to infer the power consumption of individual electrical loads by analyzing the aggregate power signal taken from a single-point measurement. Most existing NILM solutions are offline methods that do not allow the end-user to get real-time feedback on his energy consumption. In this paper, we present a near real-time NILM solution based on multi-label classification and multi-output regression. We use the multi-label classifier to predict the state of each load and use the multi-output regressor to estimate the disaggregated active power consumptions. We test our method using a publically available dataset of real power measurements. Performance results show that the proposed near real-time method can accurately estimate the energy consumption of the targeted loads with an average relative energy error of $1.55 \%$
\end{abstract}

Keywords-NILM, Load Disaggregation, Multi-label Classification, Multi-output Regression, Energy Estimation, Smart Meters.

\section{INTRODUCTION}

The worldwide increase in energy demand and climate change has resulted in new technologies which aim to reduce the use of fossil fuels as well as reducing the overall energy consumption. If actions are not undertaken, the $\mathrm{CO} 2$ emissions will double by 2050 [1]. Residential buildings consume up to $40 \%$ of total energy [1]. Studies show that consumers don't know the necessary actions which will reduce their energy bill [2]. Furthermore, $55.2 \%$ of people do prefer reducing the usage of inefficient appliances while only $11.7 \%$ prefer replacing their old appliances. This highlights the importance of providing relevant feedback information to the consumer on his energy consumption. The most common type of feedback is provided through energy bills (e.g., KWatt/hour) [2] which does not provide detailed information to the user. The effect of energy feedback on household consumption is covered in detail in [3]. Furthermore, providing appliance-level consumption information can result in more than $12 \%$ of energy savings [4] [5] [6].

Non-intrusive load monitoring (NILM) also called load or energy disaggregation aims to infer the energy consumption of single appliances from the aggregate energy use measured at the power source interface [7]. One instrumented point is sufficient to get the energy consumption of each appliance. Intrusive load monitoring (ILM) on the other hand uses one measuring system for each appliance which has the advantage to be more accurate but is more expensive and difficult to deploy at a large scale. This is why NILM is preferred when it comes to load disaggre-

Manuscript received March 23, 2021; revised September 29, 2021.

S. Sahrane and M. Haddadi are with the Ecole Nationale Polytechnique (e-mail: selim.sahrane@g.enp.edu.dz, mourad.haddadi@g.enp.edu.dz).

Digital Object Identifier (DOI): 10.53907/enpesj.v1i1.15 gation [8]. Each type of appliance or electrical load is different in the way it consumes electricity due to its internal circuitry and therefore has what is called "an appliance signature" [7]. A NILM system will typically rely on machine learning and signal processing techniques in order to extract features from each appliance's signature and to disaggregate the total energy signal by identifying different signatures. Most of the existing disaggregation approaches are offline methods [9], meaning that they use the entire dataset or day measurements before inferring the consumption of each appliance. This translates into a very low frequency of feedback that does not allow the consumer to take actions in real or near-real time. Real-time or near real-time disaggregation information is needed for the consumer in order to reduce his consumption for more than $9.2 \%$ [4] [5]. Zeifman [10] proposed six requirements for a load disaggregation system to be practical with the existing smart meter technology:

1. A sampling rate of $1 \mathrm{~Hz}$ : most smart meters use a $1 \mathrm{~Hz}$ sampling rate. The sampling frequency affects the feature extraction process and hence the NILM should be designed to work with $1 \mathrm{~Hz}$ data.

2. Accuracy: for an acceptable user experience the system should have a minimum accuracy of $80-90 \%$.

3. Easy configuration: minimum training or no training (i.e., unsupervised) and capability to adapt to new appliances and discard old ones.

4. Near real-time feedback: the system is able to give feedback on the energy use of each appliance in a minimum time interval.

5. Robustness: the ability to detect a large number of appliances (e.g., more than 20 devices).

6. Multi-type appliance recognition: some types of appliances are trickier to detect than others, light dimmers which do not have a finite sate of consumption are more 
difficult to identify than multi-sate appliances like dishwashers. A practical NILM should be able to detect all types of appliances.

These requirements are extensively used among the NILM community and are used as a reference to evaluate load disaggregation methods and there is still no complete solution that satisfies all the six requirements. The fourth requirement (i.e., near realtime capability) is not largely addressed in the NILM literature and this is what motivated us to work on this issue.

In [11] an unsupervised near real-time solution is proposed. This solution is based on the use of low-frequency features (i.e., reactive and active power) as well as high-frequency features (i.e., transients). A clustering algorithm and a manual labeling procedure are used to construct an appliance signature database. The advantage of this solution is that it is unsupervised. However, some features like transients cannot be obtained with existing smart meters. In [12] a practical implementation of a spectral decomposition-based real-time NILM solution is proposed. The authors use active power and voltage measurements obtained at a frequency of $1 \mathrm{~Hz}$. This method shows good results but has a high implementation cost due to the complexity of the used method. In [13], the author describes a NILM system able to perform disaggregation on a low-cost embedded processor in real-time using low-frequency sampling data. The method uses a super-state hidden Markov model and a Viterbi algorithm variant which preserves dependencies between loads. This approach is not scalable to a large number of appliances. In [14], a particle-based distribution truncation method is proposed. This solution uses $1 \mathrm{~Hz}$ measurements and has the ability to run in real-time. This approach presents good performance but has a high implementation cost. In fact, the authors implemented their solution on an Intel Core i7-2600 with 8GB of random access memory. In [15], a method based on particle filtering is proposed. This method uses $1 \mathrm{~Hz}$ measurements and is capable of running in real-time. For the implementation, it is reported that the algorithm can work in real-world applications on low-cost hardware such as a Raspberry Pi. In this work, we explore if the power consumptions of a given household's electrical loads can be accurately estimated in near real-time. Meaning that our NILM method should predict the current disaggregated power of each load for each aggregate power sample input.

The remaining of this paper is organized as follows: in section II., we formulate the load disaggregation problem, in section III., the proposed method is described in detail. In section IV., results are presented and a discussion is made. Finally, a conclusion is given in section V..

\section{Problem Formulation}

The aim of NILM is to separate each load's signal $S_{j}(t)$ from the aggregate signal $S_{a g g}(t)$ to then find the energy consumption of each load. The aggregate signal $S_{a g g}(t)$ is expressed as:

$$
S_{\text {agg }}(t)=\sum_{j=1}^{M} S_{j}(t)+e(t)
$$

With $M$ representing the number of loads and $e(t)$ a error term that accounts for unwanted noise. Fig. 1 shows how each ap- pliance's Signal $S_{j}(t)$ contributes to form the aggregate signal $S_{a g g}(t)$.
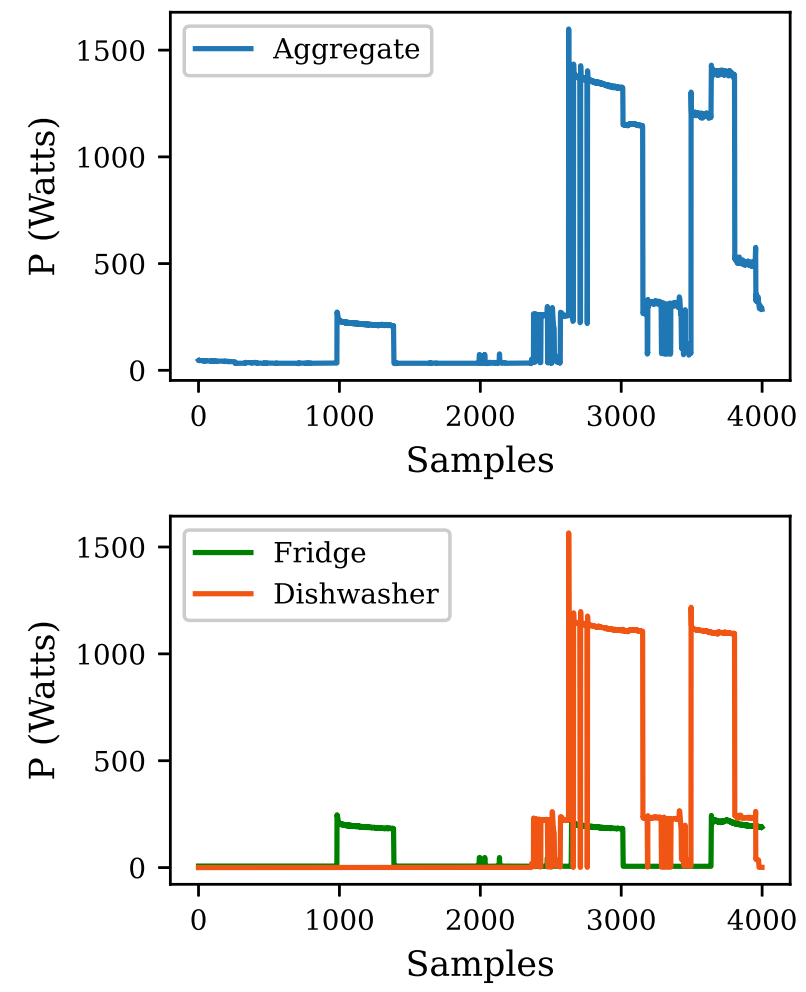

Fig. 1: Top figure shows the aggregate power signal and down figure shows the ground truth of each load.

\section{PROPOSED METHOD}

Our disaggregation method combines a multi-label classification algorithm with a multi-output regression algorithm as shown in Fig. 2. The classification step serves to predict the state of each load (ON/OFF), and the regression method returns the power consumption of each load in a near real-time fashion. More specifically, for each single aggregate power measurement, our method predicts the corresponding disaggregated power values for each load.

\section{A. Multi-label Classification}

We choose a multi-label classification approach because it is more appropriate for the load disaggregation problem. In general, multiple loads can be operating concurrently in a household, which makes their identification challenging. In a classification context, each load is represented by a unique class/label. A multi-label classification approach allows the association of multiple labels to one data instance thus, permitting to account for cases where more than one load is operating. Formally, given a set of labels $Y$, each data instance $x$ is associated with a subset $l \subset L$, with $L$ the power set of $Y$. Two types of multi-label classification methods exist, namely, problem transformation methods and algorithm adaptation methods [16]. Problem transformation methods transform the multi-label classification problem into multiple binary classification problems. Algorithm adaptations modify an existing multi-class algorithm to support multi-label classification. In this work, we use a random forest classifier algorithm implementation adapted to support multilabel classification. The random forest algorithm [17] is an 


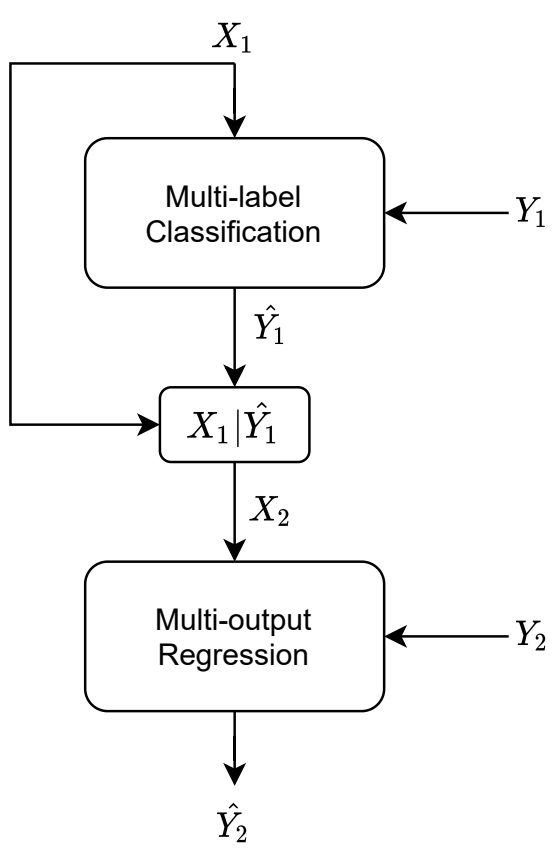

Fig. 2: Block diagram of the proposed method.

ensemble method that grows multiple decision trees on various sub-samples of the dataset and then averages the predictions to improve the predictive accuracy and control over-fitting. We use the multi-label classification to map each aggregate power sample $x_{1 i} \in X_{1}$ to a label subset $l$, with $X_{1}$ representing a vector of $N$ active power measurements. The ground truth label subset corresponding to each $x_{1 i}$ is found in the labels matrix $Y_{1}=\left[y_{1 i}, \ldots, y_{1 N}\right]^{T}$ with $y_{1 i}$ an M-binary vector containing the ON/OFF state of the $M$ loads. $\hat{Y}_{1}$ represents the predicted states of the M loads given $X_{1}$ as input to the classifier, as shown in Fig. 2.

\section{B. Multi-output Regression}

The goal of multi-output regression is to predict more than two numerical values given an input instance. As for multi-label classification, we find problem transformation methods and algorithm adaptation methods for solving the multi-output problem. An in-depth review of multi-output regression approaches is found in [18]. In this work, we use a problem transformation approach that consists of performing a separate regression for each target. Treating each target load independently is possible because the power consumptions of each load are mutually independent. The feature matrix $X_{2}$ is built using the predictions $\hat{Y}_{1}$ and the aggregate power values $X_{1}$ as shown in Fig. 2. The ground truth power trace of each load $P_{j}=\left[p_{1}, \ldots, p_{N}\right]^{T}$ is contained in the matrix $Y_{2}=\left[P_{1}, \ldots, P_{M}\right]$. To find the coefficients of our model, we use Ridge regression [19]. Unlike linear regression, which estimates the model's coefficients by minimizing the residual sum of squares between the observed targets in the data and the targets predicted by linear approximation, Ridge regression minimizes a penalized residual sum of squares. We choose to use Ridge regression because, as mentioned in [19], when using multiple independent variables, and if these variables are not perfectly uncorrelated, the residual sum of squares method has a high probability of giving unsatisfactory results. In our case, the aggregate power samples $X_{1}$ and the predicted states of each load $\hat{Y}_{1}$ are more or less correlated depending on the average consumption of each load. Because switching a load ON/OFF translates into a high/low state which increases/decreases the aggregate power.

\section{Data}

We used The REDD dataset [20]. This dataset is largely used by NILM researchers, it contains sensor measurements collected from 10 households. Also, it provides low frequency aggregate measurements as well as ground truth measurements. We considered Household 1 which contains active power of ground truth and aggregate data measured over a period of 8 days. We used $80 \%$ of the signal for training and $20 \%$ for testing. To compare our results with [15], we targeted the same appliances which are, fridge, oven, washing dryer, dishwasher, kitchen outlet, and microwave.

\section{RESUlTS AND Discussion}

The field of NILM lacks standard (or commonly adopted) metrics for the evaluation of the algorithms, making fair comparison difficult [8]. To evaluate the results of our approach, we use the F1-score (3) and the relative energy error (8). To compare our results with [15], we use the accuracy $A c c$ (2) and the normalized mean square error $N R M S E$ (6).

$$
\begin{gathered}
A c c=\frac{T P+T N}{T P+F N+T N+F P} \\
\text { F1-score }=\frac{2 \times P r \times R}{P r+R} \\
P r=\frac{T P}{T P+F P} \\
R=\frac{T P}{T P+F N}
\end{gathered}
$$

The true positive parameter $T P$ represents the number of samples that have been correctly classified or, more precisely, the power quantity correctly assigned to that device. The falsepositive parameter $F P$ represents the number of samples that have been incorrectly classified or, more precisely, the power quantity incorrectly assigned to that device. The false-negative parameter $F N$ represents the number of samples that should be but have not been classified or, more precisely, the power quantity that should have been assigned to that device but has been assigned to another or has not been assigned at all. The precision parameter $(\mathrm{Pr})$ measures the portion of power samples that have been correctly classified among the power samples assigned to a given device. The recall parameter $(R)$ measures what power portion of a given device is correctly classified in general, also considering the samples that would belong to that device but have been wrongly assigned to another or not assigned at all. Therefore, the accuracy $A c c$ measures how well each appliance is detected and the F1-score combines the results obtained through the precision and recall analysis.

$$
N R M S E=\frac{R M S E}{\bar{X}_{1}}
$$


Table. I

PERFORMANCE RESULTS FOR EACH LOAD.

\begin{tabular}{lll}
\hline Load & F1-score (\%) & Energy error (\%) \\
\hline Fridge & 96.95 & 3.93 \\
Oven & 84.71 & 0.17 \\
Dishwasher & 77.38 & 3.24 \\
Kitchen Outlet & 85.62 & 0.39 \\
Washing Dryer & 78.34 & 1.26 \\
Microwave & 89.87 & 0.33 \\
\hline
\end{tabular}

Table. II

OVERALL PERFORMANCE RESULTS OF THE PROPOSED NILM METHOD.

\begin{tabular}{lll}
\hline Macro-F1 (\%) & Micro-F1 (\%) & Average Error (\%) \\
\hline 85.48 & 91.88 & 1.55 \\
\hline
\end{tabular}

$$
\begin{gathered}
R M S E=\sqrt{\frac{\sum_{i=1}^{N}\left(\hat{x}_{1 i}-x_{1 i}\right)^{2}}{N}} \\
\text { Energy-Error }_{j}=\frac{\left|\hat{E}_{j}-E_{j}\right|}{E_{j}}
\end{gathered}
$$

With $\hat{E}_{j}$, the estimated energy consumption for the $j^{t h}$ load and $E_{j}$ is the actual energy consumed by the load.

Table. I shows the evaluation results of our method. We obtain the best classification performance for the fridge with an F1-score $=96.95 \%$. This is because the refrigerator has a less complexe load signature in comparison to other loads. Also, the refrigerator has the highest number of working cycles, thus, allowing the classifier to learn to detect it in different loads combinations scenarios. The worst classification performance is obtained for the dishwasher with an F1-score $=77.38 \%$. We found that the "wash and drain" cycle of the dishwasher consumes almost the same power as the refrigerator and, because we only use the power as a feature, the classifier can't discriminate between them. In this case, the classifier will often predict the refrigerator as it is the most populated class compared to the dishwasher. The proposed near real-time method can detect loads that work simultaneously as shown in Fig. 3. We found in our data thirty-nine different load combinations and up to four loads working simultaneously.

Table. III

PERFORMANCE COMPARISON BETWEEN OUR METHOD AND THE PALDI METHOD [15].

\begin{tabular}{lll}
\hline \multirow{2}{*}{ Load } & \multicolumn{2}{c}{ Accuracy (\%) } \\
\cline { 2 - 3 } & Our method & PALDi \\
\hline Fridge & 98.4 & 78.86 \\
Oven & 99.91 & 99.09 \\
Dishwasher & 98.57 & 77.12 \\
Kitchen Outlet & 96.72 & 98.32 \\
Washing Dryer & 99.63 & 99.53 \\
Microwave & 99.76 & 88.33 \\
\hline Total & 98.83 & 90.21 \\
NRMSE & 0.65 & 2.96 \\
\hline
\end{tabular}

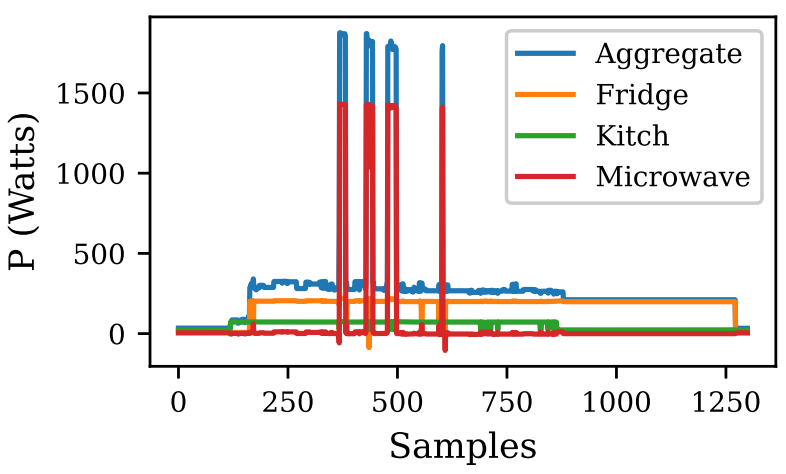

Fig. 3: Figure showing the disaggregation result obtained using our method. The power signals of the fridge, kitchen outlet, and microwave are disaggregated from the aggregate power signal.

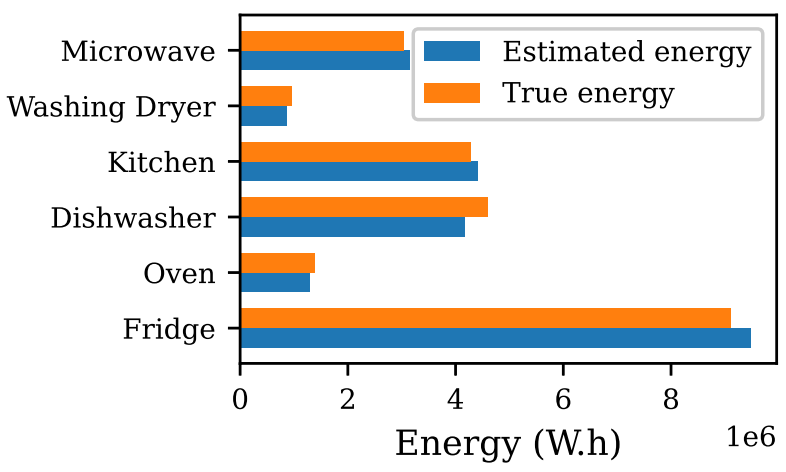

Fig. 4: Comparison between the estimated energy by our method for each load, and the corresponding actual energy consumption.

Concerning the energy estimation performance, we found that, in our case, good classification performance doesn't always result in good power/energy estimation performance. For instance, the refrigerator which is the most accurately classified load has the highest energy estimation error as shown in Table. I. High power spikes which occur when the refrigerator's compressor starts working can attain up to ten times its average power consumption. These values are difficult to predict because they don't have consistent measurement values in the dataset due to the low temporal resolution of the data. Fig. 4 shows a bar plot of the estimated energy and the actual consumed energy. Table. II gives the overall performance of the proposed method. Table III shows the comparison of the results of our method with the PALDi method [15]. We observe that the accuracy is higher for all loads except for the kitchen outlet. We also obtained a lower energy estimation error as measured with the NRMSE.

\section{CONCLUSION}

In this paper, we presented a near real-time load disaggregation method based on multi-label classification and multi-output regression. We used a multi-label classifier to predict the ON/OFF state of each load from the aggregate active power signal and a multi-output regression to estimate the power consumption of each load. The obtained results showed that our method disaggregates loads' energy consumption with low relative energy error. Using only the active power as a feature doesn't allow to differentiate between loads that consume the same power. A compromise exists between NILM feedback frequency and 
disaggregation performance. Increasing NILM feedback frequency translates into decreasing the amount of available data for NILM prediction, thus, reducing the discriminative capability of extracted features. Also, using high-frequency data may be more adapted for the near real-time NILM problem but at the expense of higher implementation costs.In the future, we will work on the hardware implementation of our method and test it on several households.

\section{REFERENCES}

[1] U. Berardi, "A cross-country comparison of the building energy consumptions and their trends," Resources, Conservation and Recycling, vol. 123, pp. 230-241, 2017. https://doi.org/10.1016/j.resconrec.2016.03.014

[2] N. J. Nunes, L. Pereira, F. Quintal, and M. Berges, "Deploying and evaluating the effectiveness of energy eco-feedback through a low-cost nilm solution," in Proceedings of the 6th International Conference on Persuasive Technology, 2011, pp. 2-5.

[3] C. Fischer, "Feedback on household electricity consumption: a tool for saving energy?" Energy efficiency, vol. 1, no. 1, pp. 79-104, 2008. https://doi.org/10.1007/s12053-008-9009-7

[4] K. C. Armel, A. Gupta, G. Shrimali, and A. Albert, "Is disaggregation the holy grail of energy efficiency? the case of electricity," Energy Policy, vol. 52, pp. 213-234, 2013. https://doi.org/10.1016/j.enpol.2012.08.062

[5] K. Ehrhardt-Martinez, K. A. Donnelly, S. Laitner et al., "Advanced metering initiatives and residential feedback programs: a meta-review for household electricity-saving opportunities." American Council for an Energy-Efficient Economy Washington, DC, 2010.

[6] S. Ahmadi-Karvigh, B. Becerik-Gerber, and L. Soibelman, "A framework for allocating personalized appliance-level disaggregated electricity consumption to daily activities," Energy and Buildings, vol. 111, pp. 337-350, 2016. https://doi.org/10.1016/j.enbuild.2015.11.029

[7] G. W. Hart, "Nonintrusive appliance load monitoring," Proceedings of the IEEE, vol. 80, no. 12, pp. 1870-1891, 1992. https://doi.org/10.1109/5.192069

[8] C. Nalmpantis and D. Vrakas, "Machine learning approaches for non-intrusive load monitoring: from qualitative to quantitative comparation," Artificial Intelligence Review, pp. 1-27, 2018. https://doi.org/10.1007/s10462-018-9613-7

[9] S. Barker, S. Kalra, D. Irwin, and P. Shenoy, "Nilm redux: The case for emphasizing applications over accuracy," in NILM-2014 workshop. Citeseer, 2014.

[10] M. Zeifman, "Disaggregation of home energy display data using probabilistic approach," IEEE Transactions on Consumer Electronics, vol. 58, no. 1, pp. 23-31, 2012. https://doi.org/10.1109/TCE.2012.6170051

[11] T. Bernard and M. Marx, "Unsupervised learning algorithm using multiple electrical low and high frequency features for the task of load disaggregation," in Proceedings of the 3rd International Workshop on NILM, Vancouver, BC, Canada, 2016, pp. 14-15.

[12] S. Welikala, N. Thelasingha, M. Akram, P. B. Ekanayake, R. I. Godaliyadda, and J. B. Ekanayake, "Implementation of a robust real-time non-intrusive load monitoring solution," Applied Energy, vol. 238, pp. 1519-1529, 2019. Implementation of a robust real-time non-intrusive load monitoring solution

[13] S. W. Makonin, "Real-time embedded low-frequency load disaggregation," Ph.D. dissertation, Applied Sciences: School of Computing Science, 2014.
[14] Y. F. Wong, T. Drummond, and Y. Şekercioğlu, "Real-time load disaggregation algorithm using particle-based distribution truncation with state occupancy model," Electronics Letters, vol. 50, no. 9, pp. 697-699, 2014. https://doi.org/10.1049/el.2013.3967

[15] D. Egarter, V. P. Bhuvana, and W. Elmenreich, "Paldi: Online load disaggregation via particle filtering," IEEE Transactions on Instrumentation and Measurement, vol. 64, no. 2, pp. 467-477, 2015. https://doi.org/10.1109/TIM.2014.2344373

[16] B. Buddhahai, W. Wongseree, and P. Rakkwamsuk, "A nonintrusive load monitoring system using multi-label classification approach," Sustainable cities and society, vol. 39, pp. 621-630, 2018. https://doi.org/10.1016/j.scs.2018.02.002

[17] T. K. Ho, "Random decision forests," in Proceedings of $3 r d$ international conference on document analysis and recognition, vol. 1. IEEE, 1995, pp. 278-282. https://doi.org/10.1109/ICDAR.1995.598994

[18] H. Borchani, G. Varando, C. Bielza, and P. Larranaga, "A survey on multi-output regression," Wiley Interdisciplinary Reviews: Data Mining and Knowledge Discovery, vol. 5, no. 5, pp. 216233, 2015. https://doi.org/10.1002/widm.1157

[19] A. E. Hoerl and R. W. Kennard, "Ridge regression: Biased estimation for nonorthogonal problems," Technometrics, vol. 12, no. 1, pp. 55-67, 1970. https://doi.org/10.2307/1271436

[20] J. Z. Kolter and M. J. Johnson, "Redd: A public data set for energy disaggregation research," in Workshop on Data Mining Applications in Sustainability (SIGKDD), San Diego, CA, vol. 25, no. Citeseer, 2011, pp. 59-62.

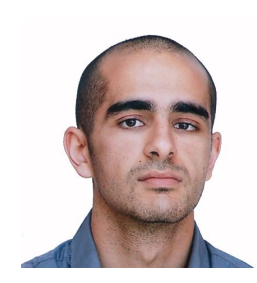

Selim Sahrane was born in Algiers, Algeria, in 1989. He received the M.Sc. degree in electronics instrumentation engineering from the USTHB, Algiers, Algeria, in 2013. Currently, he is a Ph.D. student at the Ecole Nationale Polytechnique, Algiers, Algeria. His research interests include intelligent monitoring systems, applied machine learning, and energy management systems.

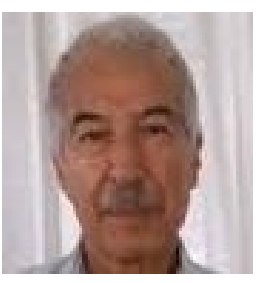

Mourad Haddadi received a Ph.D. degree in electronics from the Ecole Nationale Polytechnique in Algiers, Algeria. He is currently a Senior Researcher and Head of the Electronics Department at the Ecole Nationale Polytechnique. He was engaged in renewable energies and solar electricity for more than 30 years. His research and development tasks mainly relate to photovoltaic applications, solar energy, photovoltaic systems and devices, and the most promising applications in renewable energies. $\mathrm{He}$ is an expert and reviewer in many journals. He is the author or co-author of more than 60 publications. 